\title{
On Abnormal Vibration and Noise of Small Low Speed Wind Tunnel
}

\author{
Satoru Kudo and Kunihiko Ishihara
}

\begin{abstract}
When a small low speed wind tunnel installed in a university was operated, a large vibration occurred. Solving this problem, the vibration at the diffusion cave with large vibration and noise around the diffusion cave were measured. As a result, it was clarified that this vibration and noise were due to karman vortex generated from the radiator installed in the diffusion cave. The sound field in the diffusion cave was firstly excited, after that the resonance was occurred
\end{abstract}

Index Terms - Aerodynamic acoustics, Small low speed wind tunnel, Resonance, Self-sustained tone, Baffle plate

\section{INTRODUCTION}

Figure 1 shows a small low speed wind tunnel installed in a university. When it was operated, the abnormal vibration of $135 \mathrm{~Hz}$ was occurred and a large vibration and noise that could be felt on the 4th and 5th floors of the building, which became a problem. Therefore, in order to solve this problem, the vibration at the diffusion cave and radiator with large vibration and the noise around them were measured. As shown in figure 1, the radiator is composed of the tube bank and heat exchange is done. The cross section of the diffusion cave changes from a circular to a rectangular with going downstream. The radiator part has a rectangular cross section. When the fan is operated, a flow is generated and it flows into the radiator through the diffusion cave. At that time, an alternating vortex is generated behind the tube bank. The shedding frequency of this vortex is proportional to a flow velocity. On the other hand, since the radiator and the part of cavity forms the sound field, it exists an air column resonance frequency. This frequency is determined by the sound velocity, the size of the sound field and the boundary condition. Thus, the resonance phenomenon occurs at a certain wind speed.

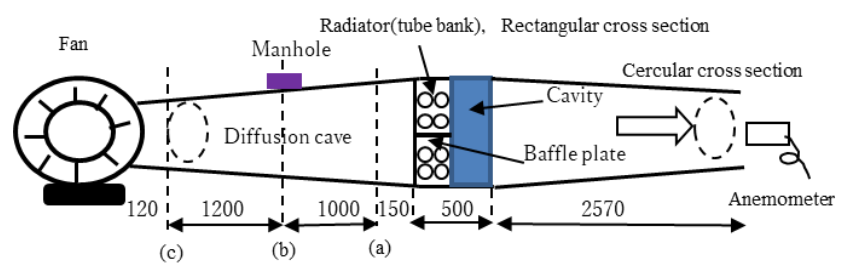

Fig.1 Outline of small low speed wind tunnel

Satoru Kudo, Department of Health and Welfare, Tokushima Bunri University, Shido, Sanuki-city, Kagawa, Japan, +81878945111.

Kunihiko Ishihara, Department of Health and Welfare, Tokushima Bunri University, Shido, Sanuki-city, Kagawa, Japan, $+81878945111$
Therefore, it is presumed that the abnormal noise is occurred by the resonance. In addition, it is presumed that the abnormal vibration at the diffusion cave and the radiator is due to the vibration force generated by the sound or vortex resonating with the structures.

This kind of problem has been studied by many authors ${ }^{(1)}$ $\sim(12)$ and useful countermeasures have been proposed ${ }^{(13) \sim(14)}$.

In this paper, the measurements will be performed in order to clarify the reason of these and their results are shown, and the validity of presume will be clarified. As a result, as expected, it became clear that these vibration and noise are caused by the resonance on the diffusion cave. The sound field in the diffusion cave was firstly excited, after that the resonance was occurred.

\section{SURVEY METHODS}

\section{A. Survey items and methods}

In order to clarify the cause of the abnormal vibration and noise, following items were surveyed.

(1) The relationship the wind speed and the vibration/ noise, and presence or absence of air column resonance by the karman vortex.

(2) The frequency and its modes at the abnormal vibration.

(3) The natural frequency and its modes (Experimental modal analysis).

(4) The acoustic mode inside the diffusion cave.

For (1), the wind speed was increased from $20 \mathrm{~m} / \mathrm{s}$ every 10 $\mathrm{m} / \mathrm{s}$, and after statically determinate, the vibration and the noise were measured. When the vibration suddenly increased, the wind speed was finely changed for measurement.

For(2) and for(3), if resonance occurs, since the frequency and its mode at abnormal vibration coincide with the natural frequency and the vibration mode. Then presence or absence of the air column resonance can be known by measuring these data.

For(4), if the natural frequency matches $135 \mathrm{~Hz}$ of the problem, it can be clarified that the sound field of diffusion cave is in resonance.

The measurement results of each of those items are shown in the next chapter.

\section{SURVEY RESULTS AND CONSIDERATIONS}

\section{A. Relation between wind speed and vibration amplitude}

Figure 2(a) shows the relationship between the wind speed and the vibration of the diffusion cave, and figure 2(b) shows the relationship between the wind speed and the noise at a point where $0.5 \mathrm{~m}$ away from it. As shown in figure 2(a) and 2(b), when the wind speed at the exit of duct is smaller than 48 $\mathrm{m} / \mathrm{s}$, the vibration and noise are absent absolutely, and they increase slightly at $49 \mathrm{~m} / \mathrm{s}$ and increase rapidly at $50 \mathrm{~m} / \mathrm{s}$. The 
frequency of the karman vortex at this time matches $135 \mathrm{~Hz}$ which is the frequency of the problem, since, the gap flow velocity of tube bank is $U \mathrm{~g}=15.8 \mathrm{~m} / \mathrm{s}$ (calculated from the cross-sectional area ratio of the radiator part and outlet), Strouhal number is $\mathrm{St}=0.22$ (from the square array of $T / d=$ $L / d=2.0)^{(15)}$, the tube diameter $\mathrm{d}=25.4 \mathrm{~mm}$, so $f_{v}=0.22 U \mathrm{~g} / \mathrm{d}$ $=0.22 \times 15.6 \times / 0.00254=135 \mathrm{~Hz}$. Looking at the noise measurement results in figure 2 (b), it can be seen that the sound pressure level increases by following the six power law which is the characteristics of the aerodynamic noise with dipole. It can be known by increasing of $18 \mathrm{~dB} / \mathrm{DV}$ (DV: Double wind speed) when the wind speed is from $20 \mathrm{~m} / \mathrm{s}$ to 48 $\mathrm{m} / \mathrm{s}$.

In general, the aerodynamic noise is followed by Eq.(1).

$$
I \propto U^{6} \quad(I: \text { Sound intensity, } U: \text { Wind speed })
$$

Then it can be seen that aerodynamic noise appears in figure 2(b). In addition, when the wind speed is larger than $48 \mathrm{~m} / \mathrm{s}$, the sound pressure level increases rapidly, and the sound pressure level reaches about $100 \mathrm{~dB}$ at $50 \mathrm{~m} / \mathrm{s}$. And when the wind speed is further increased, the sound pressure level keeps a large constant level. At this time, the frequency is also kept constant of $135 \mathrm{~Hz}$. The phenomenon like this is called "Lock in", a self-sustained mechanism is created and it becomes a very dangerous state, the wind tunnel must be shut down immediately.

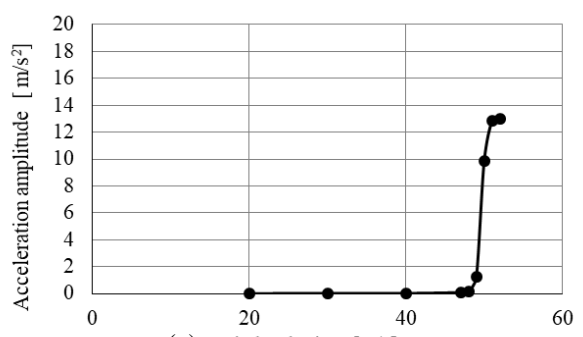

(a) Wind velocity $[\mathrm{m} / \mathrm{s}]$

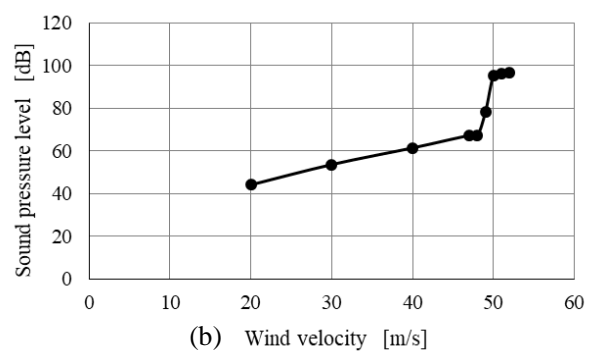

Fig.2 Relationship of velocity and vibration or noise.

\section{B. Vibration modes at occurrence of abnormal vibration}

Figure 3 shows the vibration mode when the abnormal vibration occurs. The three positions of cross sections ((a): $150 \mathrm{~mm}$ from the radiator side, (b):1320 $\mathrm{mm}$ from the fan side, (c): $120 \mathrm{~mm}$ from the fan side) are shown by broken lines in the longitudinal direction of the diffusion cave as shown in figure 1 . And the vibrations of radial direction were measured at 20 points in the circumferential direction. The left and right positions (a) and (c) have the same vibration mode (oval type), but the central one (b) is a petal-like mode. This is because the constraint conditions have changed due to existing a manhole in the center.

\section{Inertance of diffusion cave}

Figure 4 shows one example of the inertance of the diffusion cave obtained by using the experimental modal analysis. In addition, figure 5 shows the natural frequencies and their modes of the five positions of diffusion cave. They include three points of figute3. There is no natural frequency that exactly matches the $135 \mathrm{~Hz}$ which is the frequency of the problem $(136.25 \mathrm{~Hz}$ to be exact), but there is a natural frequency of $133.75 \mathrm{~Hz}$ close to that. Comparing figure 5 with figure 3, they almost match each other, so it can be said that it is in a state of resonance with an exciting force of $135 \mathrm{~Hz}$.

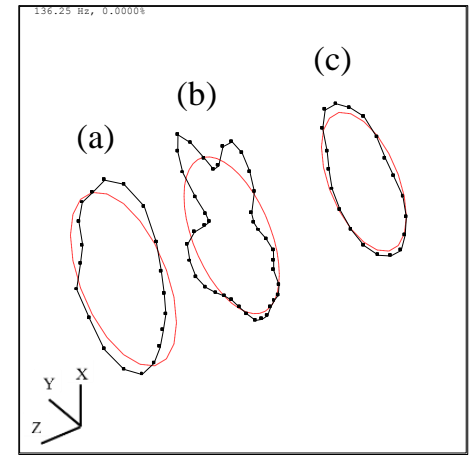

Fig.3 Vibration modes at occurrence of abnormal vibration

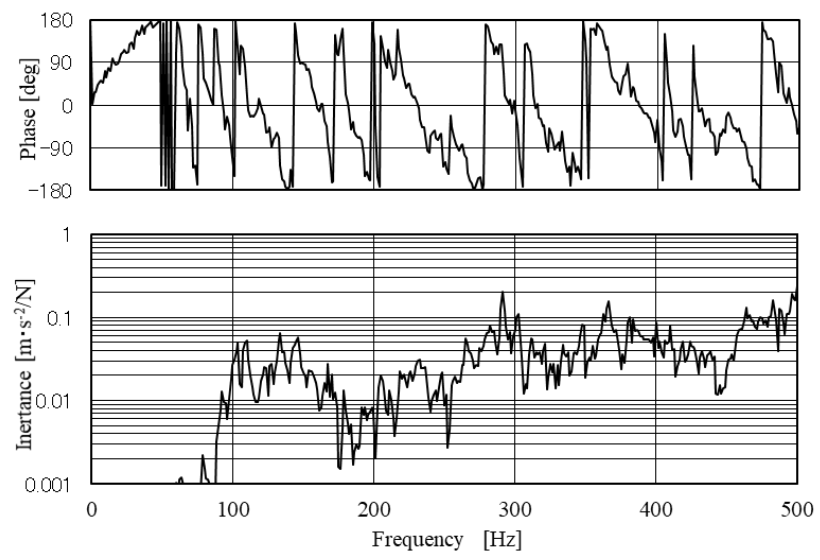

Fig.4 Inertance of the 3rd diffusion cave.

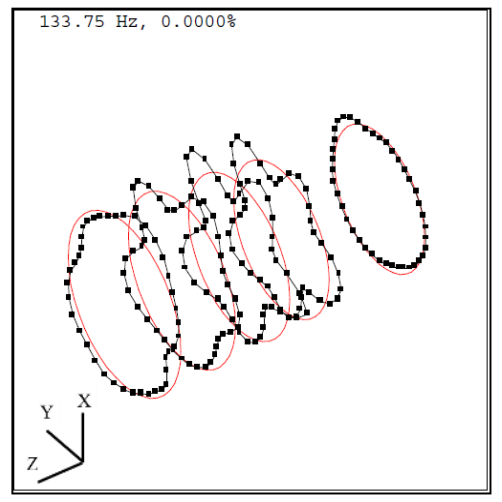

Fig.5 Natural frequency and mode 


\section{Vibration modes at occurrence of abnormal vibration}

Figure 6 shows the sound pressure spectra at each position in the longitudinal direction of the diffusion cave. Looking at this, the $135 \mathrm{~Hz}$ component can be seen near the radiator (upper part of Fig. 6), but the magnitude of this component decreases monotonously as the distance increases. From this, it can be seen that the $135 \mathrm{~Hz}$ component is not the resonance mode of the longitudinal direction of the diffusion cave. Therefore, we investigated the sound pressure in the cavity at the joint between the radiator and the diffusion cave (Figure 7). Looking at figure $7,135 \mathrm{~Hz}$ is clearly observed. Therefore, $135 \mathrm{~Hz}$ sine wave excitation was performed to obtain the acoustic modes. This is shown in figure 8. From this figure, it can be seen that the half-wavelength (1st) mode is clearly generated. In addition, when the resonance frequency of the radiator part is estimated in taking account of coupling in the longitudinal direction, $f=(\mathrm{c} / 2) \cdot\left\{\left(1 / \mathrm{L}_{x}\right)^{2}+\left(1 / \mathrm{L}_{y}\right)^{2}\right\}^{1 / 2}=131.2$ $\mathrm{Hz}$, which was close to the measured value. Here, $\mathrm{L}_{x}$ and $\mathrm{L}_{y}$ are the lengths of the longitudinal direction and the height direction, respectively. Therefore, it is concluded that the abnormal vibration of $135 \mathrm{~Hz}$ was generated by the excitation of this acoustic mode, which became the excitation source and
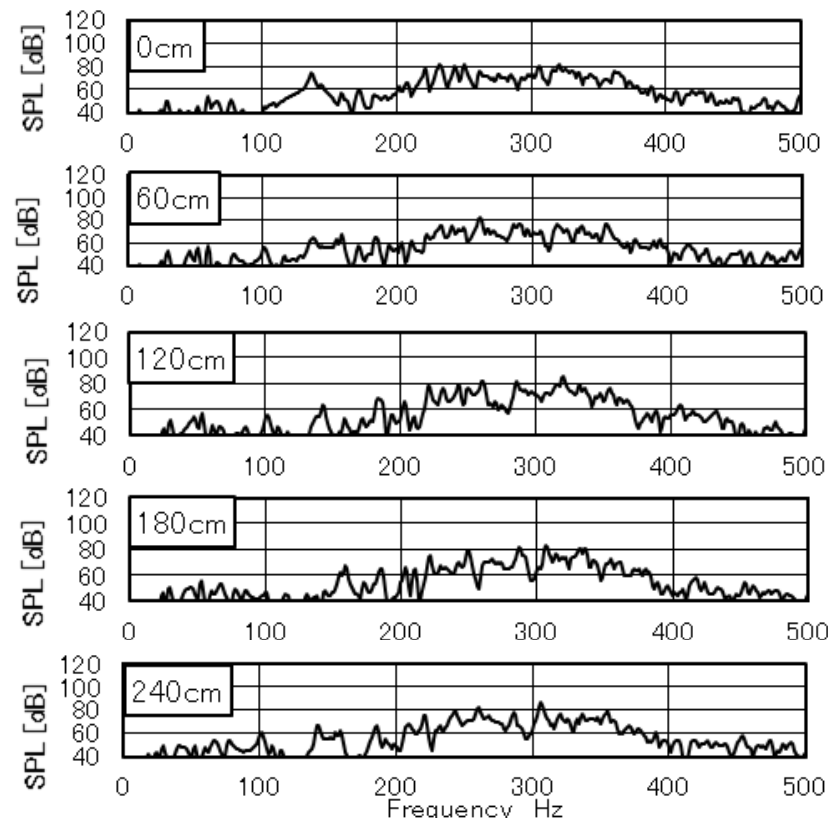

Fig.6 Sound pressure spectra measured every $60 \mathrm{~cm}$ from the radiator (longitudinal direction of the diffusion cave)
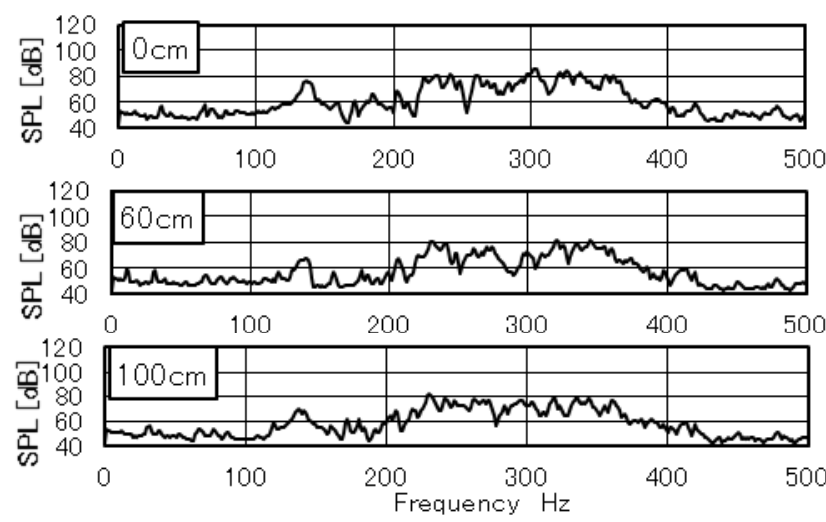

Fig.7 Sound pressure spectra measured every $60 \mathrm{~cm}$ from the top to the bottom at the center position of the radiator. resonated with the diffusion cave. Here, $c=390 \mathrm{~m} / \mathrm{s}$ $\left(\mathrm{t}=105^{\circ} \mathrm{C}, \mathrm{L}_{x}=5.5 \mathrm{~m}, \mathrm{~L}_{y}=1.5 \mathrm{~m}\right.$.

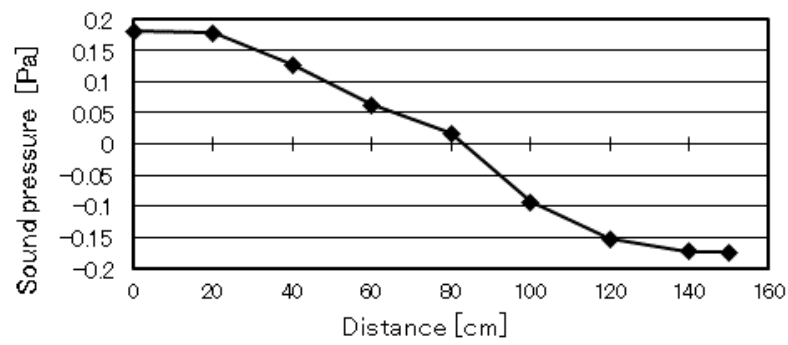

Fig. 8 Acoustic mode of $135 \mathrm{~Hz}$ component in speaker test.

\section{EVALUATION OF THE COUNTERMEASURE}

In order to prevent resonance in the first mode, a veneer plate (baffle plate) was placed in the center of the height where the particle velocity was maximum in the occurrence of abnormal noise of $135 \mathrm{~Hz}$. Figure 10 and figure 11 show the time-series waveforms and sound pressure spectrum before and after installation the baffle plate, respectively. Looking at this, it can be seen that the sound pressure is reduced by about $20 \mathrm{~dB}$ by installing the baffle plate and the insertion of baffle plate is effective for the countermeasure.
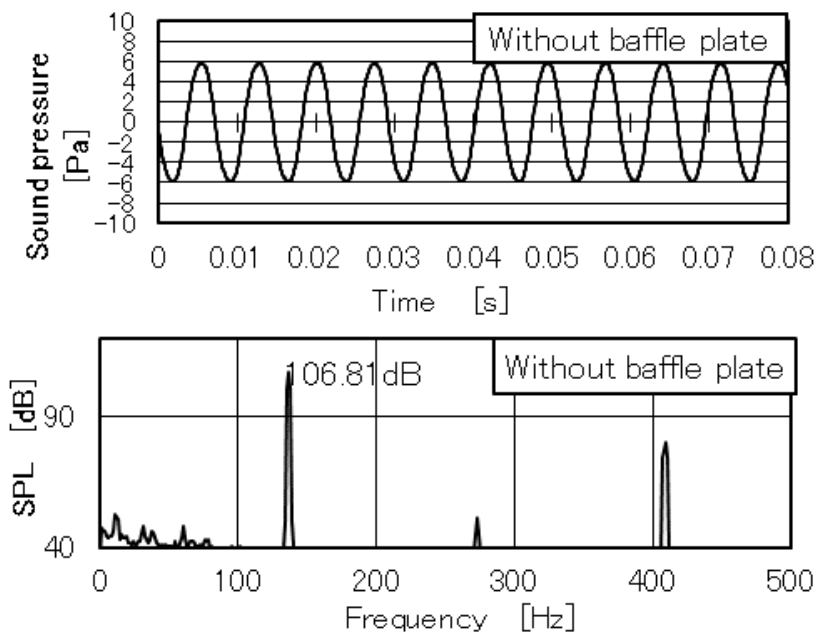

Fig.10 Sound pressure in the case of without the baffle plate.
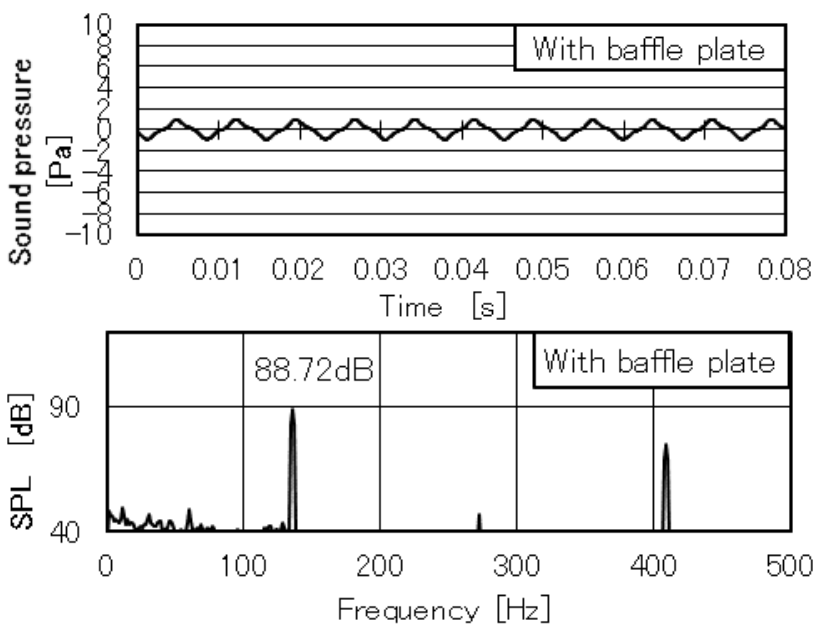

Fig.11 Sound pressure in the case of with the baffle plate. 
Therefore, an iron plate with a width of $20 \mathrm{~cm}$ was attached by temporary welding, and the effect of the baffle plate was confirmed by actually blowing the wind. Figure 12 compares the vibration results with and without the baffle plate. By installing the baffle plate, the vibration was reduced to $1 / 3$. As can be seen from this figure, when there is no baffle plate, the sound is large and the sound pressure is kept constant even if the wind speed increases, but when there is baffle plate, the sound is small and tends to decrease again as the wind speed increases. This is because the installation of the baffle plate weakens the shedding of the vortex, and the self-excitation mechanism could not be created. Therefore, the result was like a forced vibration (resonance curve). From this, the width of the baffle plate was further increased, and by installing it on the downstream side of the radiator, the abnormal vibration and the noise could be completely disappeared.

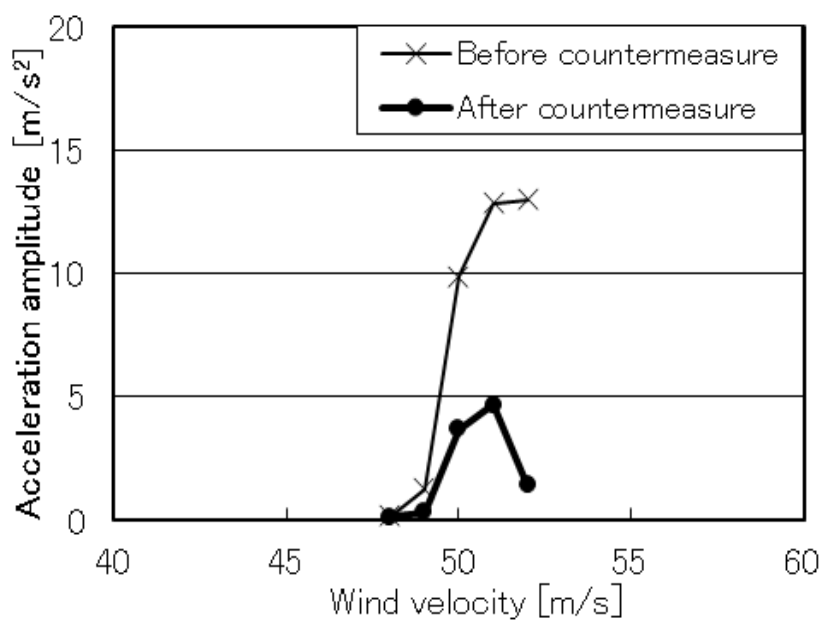

Fig.11 Comparison of amplitude of vibration with and without countermeasure.

\section{$\mathrm{V}$ CONCLUSIONS}

An abnormal vibration and noise of $135 \mathrm{~Hz}$ occurred in a small low-speed wind tunnel installed in a university, so a field survey was conducted for the purpose of investigating the cause and countermeasures. As a result, the following conclusions were obtained.

(1) The abnormal vibration of $135 \mathrm{~Hz}$ occurred at a wind speed of about $49 \mathrm{~m} / \mathrm{s}$ is due to the resonance of the diffusion cave with the exciting force which is generated by acoustic resonance of the cave with the vortex shedding.

(2) From the above cause, it is necessary to install a baffle plate to weaken the shedding of the vortex and not to create the self-excited mechanism. Actually, although it was tentative, when the baffle plate was installed on the upstream side of the radiator the vibration was reduced to $1 / 3$. From this, the width of the baffle plate was further increased, and by installing it on the downstream side of the radiator, the abnormal vibration and the noise could be completely disappeared.

\section{ACKNOWLEGEMENT}

I would deeply appreciate Professor Karashima, Professor Ochi and Mr. Hayashi who give me useful advices in measuring the sound pressure level of the small low speed wind tunnel.

\section{REFERENCES}

[1] R.C. Baird, "Pulsation-Induced Vibration in Utility Steam Generation Unit", Combust. 1954, 25, 38-44.

[2] P.R. Owen, "Buffeting Excitation of Boiler Tube Vibration," J. Mech. Eng. Sci. 1965, 7, 4, 431-439.

[3] Y.N. Chen, "Flow-induced Vibration and Noise in Tube-bank Heat Exchangers due to Von Karman Streets", J. Eng. Ind. 1968, 90, 1, 134-146.

[4] M. Funakawa, "The Acoustic Resonance in a Tube Bank". J. JSME. ,1970, 13, 348-355.

[5] J.A. Fitzpatric, "The Prediction of Flow-Induced Noise in Heat Exchanger Tube Arrays", J. Sound. Vib., 1985, 99, 425-435.

[6] R.D. Blevins, Bressler M.M. "Acoustic Resonance in Heat Exchanger Tube Bundles-Part I: Physical Nature of the Phenomena", J. Press. Vess. Technol. , 1987, 10, 275-281.

[7] D.S. Weaver, "Vortex Shedding and Acoustic Resonance In Heat Exchanger Tube Arrays", Technology for the 90's, ASME Special Publication, New York, 1993; pp.776-810.

[8] S. Ziada, Oengören, A. "Vortex Shedding in an In-Line Tube Bundle with Large Tube Spacings", J. Fluid. Struct. , 1993, 7, 6, pp.661-687.

[9] S. Ziada, "Vorticity Shedding and Acoustic Resonance of Tube Bundles", J. Braz. Soc. Mech. Sci. 2006, 28, 2, pp.186-199.

[10] F. L. Eisinger and R. E. Sullivan, "Acoustic Resonance in a Package Boiler and Its Solution A Case Study”, J. Press. Vess. Technol. 2006, 129, pp.759-762.

[11] H. Hamakawa, H. Matsue, E, Nishida and T. Fukano, "Acoustic Resonance and Vortex Shedding from Tube Banks of Boiler Plant", $J$. Fluid. Sci. Technol. 2008, 3, 6, pp.805-813.

[12] H. Hamakawa, T, Nakamura, K, Asakura, E. Nishida and E. Kurihara, "Effect of Arrangement of Tube Banks on Acoustic Resonance", O. J. Fluid. Dy. 2012, 2, pp.311-317.

[13] K. Ishihara, T. Tamehira, M. Tsujii and M. Ichimiya, "Study on a Countermeasure of Self-Sustained Tone by a Baffle Plate in Boiler Tube Banks", J. Basic. Appl. Phys. 2013, 2, Iss.3, pp.148-154.

[14] K. Ishihara and M. Nakaoka, "Study on a Countermeasure Using Walls Made of Perforated Plate for High Level Sound", In proceedings of ASME 2015 Pressure Vessels and Piping Conference Volume 4: Fluid-Structure Interaction, Boston, Massachusetts, USA, pp.19-23, July, 2015.

[15] R. D. Blevins, Flow-Induced Vibration (Second Edition),KRIEGER PUBLISHING COMPANY, MALABAR, FLORIDA, 2001, p.49

Satoru Kudo was born in 1983 in Kure city, Hiroshima Prefecture Japan, He received the B.S. degree from The Hiroshima International University in 2006. He received master's degree of medical engineering from the same University in 2008 and received the Ph.D. degree of medical engineering from the same University in 2018.

He worked in JA Yoshida General Hospital for eight years and now he has been working in Tokushima Bunri University. He teaches Biomedical engineering and has studied the field related hemodialysis.

Kunihiko Ishihara was born in 1947 in Kurashiki City, Okayama Prefecture Japan. He received the B.S. degree from Kobe University in 1969. He got a master's degree in Kobe University in 1971 and earned the Ph.D. degree in Engineering from The Osaka University in 1986.

He worked in Kawasaki Heavy Industry Co. Ltd. as an Mechanical Engineer for 33 years. After that he became a Professor of The University of Tokushima in 2004. He had been studying the vibration and noise control, above all he studied the flow induced vibration and noise problems. He has authored or co-authored over 120 technical journal and over 50 conference papers. He is a fellow of JSME (Japan Society of Mechanical Engineers) now. He is a Professor of Tokushima Bunri University. He teaches a mechanical field subjects for students. 